\title{
Carbon Nanotubes and Exotic Transport Properties
}

\author{
Tsuneya Ando \\ ${ }^{a}$ Department of Physics, Tokyo Institute of Technology, 2-12-1 Ookayama, Meguro-ku, Tokyo 152-8551, Japan
}

\begin{abstract}
A brief review is given on electronic and transport properties of carbon nanotubes mainly from a theoretical point of view. The topics include an effective-mass description of electronic states, the absence of backward scattering except for scatterers with a potential range smaller than the lattice constant, the presence of perfectly conducting channel, effects of inelastic scattering, dynamical conductivity, and a quantum correction.
\end{abstract}

Key words: graphite, carbon nanotube, impurity scattering, phonon scattering, perfect conductor, dynamical conductivity PACS: 72.10-d, 73.50-h, 73.40-h

\section{Introduction}

Carbon nanotubes $(\mathrm{CN})$ are either a metal or semiconductor, depending on their diameters and helical arrangement. This condition can be obtained based on the band structure of a two-dimensional (2D) graphite sheet and periodic boundary conditions along the circumference direction. This result was first predicted by means of a tight-binding model and was well reproduced in a $\mathbf{k} \cdot \mathbf{p}$ method or an effective-mass approximation [1]. The purpose of this paper is to give a brief review of recent theoretical study on electronic and transport properties of carbon nanotubes.

Transport properties of CNs are interesting because of their unique topological structure. There have been some reports on experimental study of transport in CN bundles [2] and ropes [3,4]. Transport measurements became possible for a single multi-wall nanotube [5-10] and a single single-wall nanotube [11-15]. Single-wall nanotubes exhibit large charging effects presumably due to nonideal contacts [16-20]. Almost ideal contacts were realized also [21].

Preprint submitted to Physica E

\section{Neutrino on Cylinder Surface}

A graphite sheet is a zero-gap semiconductor in the sense that the conduction and valence bands consisting of $\pi$ states cross at $\mathrm{K}$ and $\mathrm{K}^{\prime}$ points of the Brillouin zone, whose wave vectors are given by $\mathbf{K}$ and $\mathbf{K}^{\prime}[22]$. Electronic states near a $\mathrm{K}$ point of $2 \mathrm{D}$ graphite are described by the $\mathbf{k} \cdot \mathbf{p}$ equation [23,1]:

$$
\gamma(\boldsymbol{\sigma} \cdot \hat{\mathbf{k}}) \mathbf{F}^{K}(\mathbf{r})=\varepsilon \mathbf{F}^{K}(\mathbf{r}), \quad \mathbf{F}^{K}(\mathbf{r})=\left(\begin{array}{c}
F_{A}^{K}(\mathbf{r}) \\
F_{B}^{K}(\mathbf{r})
\end{array}\right),
$$

where $\gamma$ is the band parameter, $\hat{\mathbf{k}}=\left(\hat{k}_{x}, \hat{k}_{y}\right)$ is a wavevector operator, $\varepsilon$ is the energy, and $\sigma_{x}$ and $\sigma_{y}$ are the Pauli spin matrices. Equation (1) has the form of Weyl's equation for neutrinos.

The electronic states can be obtained by imposing the periodic boundary condition in the circumference direction $\Psi(\mathbf{r}+\mathbf{L})=\Psi(\mathbf{r})$ except for extremely thin CNs. The Bloch functions at a $\mathrm{K}$ point change their phase by $\exp (i \mathbf{K} \cdot \mathbf{L})=\exp (2 \pi i \nu / 3)$, where $\nu=0$ or \pm 1 , determined by the chiral vector $\mathbf{L}$ specifying the nanotube. Because $\Psi(\mathbf{r})$ is written as a product of the 
Bloch function and the envelope function, this phase change should be canceled by that of the envelope functions and the boundary conditions for the envelope functions are given by $\mathbf{F}^{K}(\mathbf{r}+\mathbf{L})=\mathbf{F}^{K}(\mathbf{r}) \exp (-2 \pi i \nu / 3)$.

Energy levels in $\mathrm{CN}$ for the $\mathrm{K}$ point are obtained by putting $k_{x}=\kappa_{\nu}(n)$ with $\kappa_{\nu}(n)=(2 \pi / L)[n-(\nu / 3)]$ and $k_{y}=k$ in the above $\mathbf{k} \cdot \mathbf{p}$ equation as $\varepsilon_{\nu}^{( \pm)}(n, k)=$ $\pm \gamma \sqrt{\kappa_{\nu}(n)^{2}+k^{2}}[1]$, where $L=|\mathbf{L}|, n$ is an integer, and the upper $(+)$ and lower $(-)$ signs represent the conduction and valence bands, respectively. The Hamiltonian for $\mathbf{F}^{K^{\prime}}$ for the $\mathrm{K}^{\prime}$ point is obtained by replacing $\hat{k}_{y}$ by $-\hat{k}_{y}$ and therefore the corresponding energy levels are obtained by replacing $\nu$ by $-\nu$. This shows that $\mathrm{CN}$ becomes metallic for $\nu=0$ and semiconducting with gap $E_{g}=4 \pi \gamma / 3 L$ for $\nu= \pm 1$.

An important feature is the presence of a topological singularity at $\mathbf{k}=0$. A neutrino has a helicity and its spin is quantized into the direction of its motion. The spin eigen function changes its signature due to Berry's phase under a $2 \pi$ rotation. Therefore, the wave function acquires phase $-\pi$ when the wave vector $\mathbf{k}$ is rotated around the origin along a closed contour [24,25]. The signature change occurs only when the contour encircles $\mathbf{k}=0$ but not when the contour does not contain $\mathbf{k}=0$. This topological singularity at $\mathbf{k}=0$ is the origin of the absence of backscattering in metallic carbon nanotubes as discussed below.

\section{Ideal Conductor}

In the presence of impurities, electronic states in the vicinity of $\mathrm{K}$ and $\mathrm{K}$ ' points can be mixed with each other. Therefore, we should use a $4 \times 4$ equation

$$
H \mathbf{F}=\varepsilon \mathbf{F}, \quad H=H_{0}+V, \quad \mathbf{F}=\left(\begin{array}{c}
\mathbf{F}^{K} \\
\mathbf{F}^{K^{\prime}}
\end{array}\right) .
$$

The effective potential of an impurity is written as [26]

$$
V=\left(\begin{array}{cccc}
u_{A}(\mathbf{r}) & 0 & e^{i \eta} u_{A}^{\prime}(\mathbf{r}) & 0 \\
0 & u_{B}(\mathbf{r}) & 0-\omega^{-1} e^{-i \eta} u_{B}^{\prime}(\mathbf{r}) \\
e^{-i \eta} u_{A}^{\prime}(\mathbf{r})^{*} & 0 & u_{A}(\mathbf{r}) & 0 \\
0 & -\omega e^{i \eta} u_{B}^{\prime}(\mathbf{r})^{*} & 0 & u_{B}(\mathbf{r})
\end{array}\right)
$$

where $\omega=\exp (2 \pi i / 3)$ and $\eta$ is the direction angle of $\mathbf{L}$ called chiral angle. If we use a tight-binding model, for example, we can obtain the explicit expressions for the potentials.
With the increase of the range, the off-diagonal intervalley term decreases and vanishes when the range exceeds the lattice constant, and the diagonal terms $u_{A}(\mathbf{r})$ and $u_{B}(\mathbf{r})$ become equal to each other also. This leads to the absence of the backward scattering and the realization of a perfect conductor in the presence of scatterers.

It has been proved that the Born series for backward scattering vanish identically [26]. The absence of backward scattering has been confirmed by numerical calculations in a tight binding model [27]. This unique property is the result of the topological singularity of the neutrino equation [25].

Backscattering corresponds to a rotation of the $\mathbf{k}$ direction by $\pm \pi$. In the absence of a magnetic field, there exists a time reversal process corresponding to each backscattering process. The time reversal process corresponds to a rotation of the $\mathbf{k}$ direction by $\pm \pi$ in the opposite direction. The scattering amplitudes of these two processes are same in the absolute value but have an opposite signature because of Berry's phase. As a result, the backscattering amplitude cancels out completely. In semiconducting nanotubes, on the other hand, backscattering appears because the symmetry is destroyed by a nonzero AB magnetic flux.

An important information has been obtained on the mean free path in nanotubes by single-electron tunneling experiments $[11,15]$. The Coulomb oscillation in semiconducting nanotubes is quite irregular and can be explained only if nanotubes are divided into many separate spatial regions in contrast to that in metallic nanotubes [28]. This behavior is consistent with the presence of backscattering leading to a localization of the wave function. In metallic nanotubes, the wave function is extended in the whole nanotube because of the absence of backscattering. With the use of electrostatic force microscopy the voltage drop in a metallic nanotube has been shown to be negligible [29].

Single-wall nanotubes usually exhibit large charging effects due to nonideal contacts. Contacts problems were investigated theoretically in various models and an ideal contact was suggested to be possible under certain conditions [16-20]. Recently, almost ideal contacts were realized and a Fabry-Perot type oscillation due to reflection by contacts was claimed to be observed [21].

At nonzero temperatures, lattice vibrations usually constitute the major source of electron scattering and limit the resistivity. Usually long-wavelength 
acoustic phonons are most important. These modes can be described by a continuum model [30-32]. An acoustic phonon gives rise to an effective electronphonon coupling called the deformation potential $V_{1}=g_{1}\left(u_{x x}+u_{y y}\right)$ with $u_{x x}=\left(\partial u_{x} / \partial x\right)+\left(u_{z} / R\right)$ and $u_{y y}=\partial u_{y} / \partial y$, where $R$ is the $\mathrm{CN}$ radius and $u_{x}$, $u_{y}$, and $u_{z}$ represent lattice displacements in the $x, y$, and $z$ directions, respectively. This potential appears as a diagonal term. A very rough estimation gives $g_{1} \sim 30 \mathrm{eV}$.

Phonon causes also a change in the distance between neighboring carbon atoms. This gives rise to an offdiagonal elements $V_{2}=g_{2} e^{3 i \eta}\left(u_{x x}-u_{y y}+2 i u_{x y}\right)$ with $g_{2}=(3 \alpha \beta / 4) \gamma_{0}$, where $-\gamma_{0}$ is the transfer between nearest-neighbors, $\beta=-d \ln \gamma_{0} / d \ln b, b$ is the bond length, and $\alpha$ is a quantity smaller than unity dependent on microscopic models of phonons. Usually, we have $\beta \sim 2$ [33] and $\alpha \sim 1 / 3$, which give $g_{2} \sim \gamma_{0} / 2$ or $g_{2} \sim$ $1.5 \mathrm{eV}$. This coupling constant is much smaller than the deformation potential. It corresponds to one given by Kane and Mele [34] without the reduction factor $\alpha$.

The diagonal term does not contribute to the backscattering and only the much smaller off-diagonal term has some contribution. The mean free path $\Lambda$ is estimated as $\Lambda=\left(\mu a^{2} / 3 k_{B} T \alpha^{2} \beta^{2}\right) L$, where $\mu$ is the shear modulus of $2 \mathrm{D}$ graphite and $a$ is the lattice constant. We obtain $\Lambda / L \sim 600$ at room temperature, which is larger than $1 \mu \mathrm{m}$ for thin armchair nanotubes with diameter $\sim 1.5 \mathrm{~nm}$ and increases in proportion to $L$ with $L$. This shows that a metallic $\mathrm{CN}$ is ballistic even at room temperature.

\section{Perfect Conducting Channel}

When the Fermi level moves away from the energy range where only linear bands are present, interband scattering appears because of the presence of several bands at the Fermi level. Let $r_{\bar{\beta} \alpha}$ be the reflection coefficient from a state with wave vector $\mathbf{k}_{\alpha}$ to a state with $\mathbf{k}_{\bar{\beta}} \equiv-\mathbf{k}_{\beta}$ in a $2 \mathrm{D}$ graphite sheet. Here, $\bar{\beta}$ stands for the state with wave vector opposite to $\beta$. Apart from a trivial phase factor arising from the choice in the phase of the wave function, the reflection coefficients satisfy the symmetry relation [35] $r_{\bar{\beta} \alpha}=-r_{\bar{\alpha} \beta}$. This leads to the absence of backward scattering $r_{\bar{\alpha} \alpha}=-r_{\bar{\alpha} \alpha}=0$ in the single-channel case.
Define the reflection matrix $r$ by $[r]_{\alpha \beta}=r_{\bar{\alpha} \beta}$. Then, we have $r=-{ }^{t} r$, where ${ }^{t} r$ is the transpose of $r$. In general we have $\operatorname{det}^{t} P=\operatorname{det} P$ for any matrix $P$, where $\operatorname{det} P$ is the determinant of $P$. In metallic nanotubes, the number of traveling modes $n_{\mathrm{c}}$ is always given by an odd integer and therefore $\operatorname{det}(-r)=-\operatorname{det}(r)$, leading to $\operatorname{det}(r)=0$.

By definition, $r_{\bar{\beta} \alpha}$ represents the amplitude of an out-going mode $\bar{\beta}$ with wave function $\psi_{\bar{\beta}}(\mathbf{r})$ for the reflected wave corresponding to an in-coming mode $\alpha$ with wave function $\psi_{\alpha}(\mathbf{r})$. Therefore, the vanishing determinant of $r$ shows the presence of a mode which is transmitted through the system with probability one without being scattered back. In fact, numerical calculations show that the conductance decreases with the length but remains larger than the ideal conductance for a single channel [35].

The transport may be discussed using a conventional Boltzmann equation. The transport equation is converted into the equation in terms of mean free path for each band in quasi-one-dimensional systems [36,37] and the conductivity becomes the sum of the mean free path of each band. The result shows that the Boltzmann equation gives an infinite conductivity as long as the Fermi level lies in the energy range $-1<\varepsilon L / 2 \pi \gamma<$ +1 where only the linear metallic bands are present. However, the conductivity becomes finite when the Fermi energy moves away from this energy range into the range where other bands are present.

This conclusion is quite in contrast to the exact prediction that there is at least a channel which transmits with probability unity, leading to the conclusion that the conductance is given by $2 e^{2} / \pi \hbar$ independent of the energy for sufficiently long nanotubes. The difference originates from the absence of phase coherence in the approach based on a transport equation. In fact, in the transport equation scattering from each impurity is treated as a completely independent event after which an electron looses its phase memory, while in the transmission approach the phase coherence is maintained throughout the system.

Effects of inelastic scattering can be considered in a model in which the nanotube is separated into segments with length of the order of the phase coherence length $L_{\phi}$ and the electron looses the phase information after the transmission through each segment. Figure 1 shows some examples of calculated conductance. As long as the length is smaller than or comparable to 


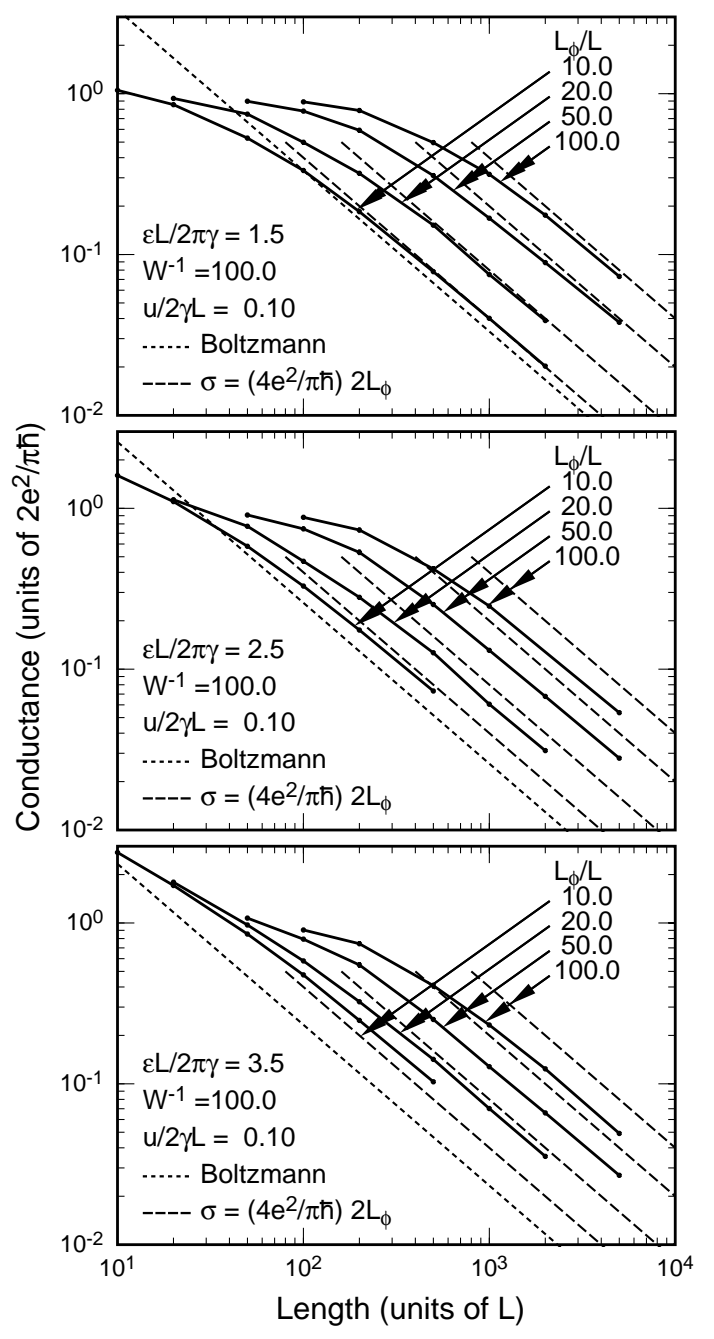

Fig. 1. The conductance in the presence of inelastic scattering as a function of the length for different values of the phase coherence length $L_{\phi}$. The number of conducting channels is 3,5 , and 7 from the top to the bottom. $W$ represents effective strength of scatterers and $u$ strength of the potential of each scatterer.

$L_{\phi}$, the conductance is close to the ideal value $2 e^{2} / \pi \hbar$ corresponding to the presence of a perfect channel. When the length becomes much larger than $L_{\phi}$, the conductance decreases in proportion to the inverse of the length. With the decrease of $L_{\phi}$, the conductance decreases and becomes closer to the Boltzmann result given by the dotted line. These results correspond to the fact that a conductivity can be defined for finite $L_{\phi}$ such that it is proportional to $L_{\phi}$ with a coefficient,

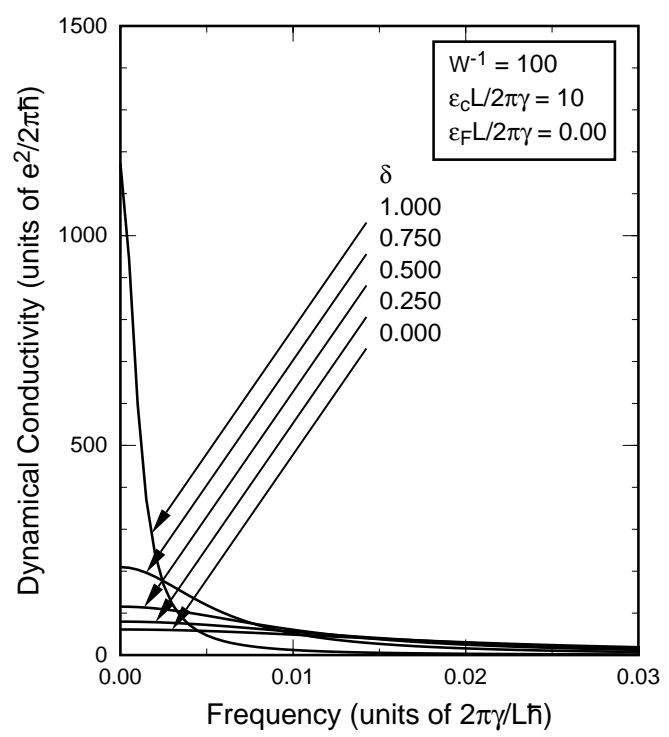

Fig. 2. Some examples of calculated dynamical conductivity in a metallic nanotube for $\varepsilon_{F}=0$. The strength of long-range scatterers is denoted by $\delta(\delta=1$ for long-range scatterers only and $\delta=0$ for short-range scatterers only).

which decreases slowly with the number of the currentcarrying channels and weakly depends on $L_{\phi}$.

\section{Dynamical Conductivity}

The dynamical conductivity $\sigma(\omega)$ is another important quantity characterizing the system and exhibits also an intriguing behavior. In fact, calculations in a self-consistent Born approximation give a Drude behavior $\sigma(\omega)=\sigma(0) /\left(1+\omega^{2} \tau_{\text {eff }}^{2}\right)$ in the limit of small frequency [38]. When the Fermi level lies in the linear metallic bands the effective relaxation time $\tau_{\text {eff }}$ becomes infinite and $\sigma(\omega) \propto \delta(\omega)$ as long as other bands are completely neglected. When effects of other bands are included, however, $\tau_{\text {eff }}$ becomes finite, leading to a finite conductivity. When the Fermi level lies in higher or lower bands, $\tau_{\text {eff }}$ becomes much smaller and close to the Boltzmann result.

Figure 2 shows some examples of calculated dynamical conductivity for the Fermi energy $\varepsilon_{F}=0$. The parameter $\delta$ describes the relative importance of scatterers with a potential range larger than the lattice constant $(\delta=1)$ and those with a range smaller $(\delta=0)$. In the absence of short-range scatterers $(\delta=1)$ the con- 


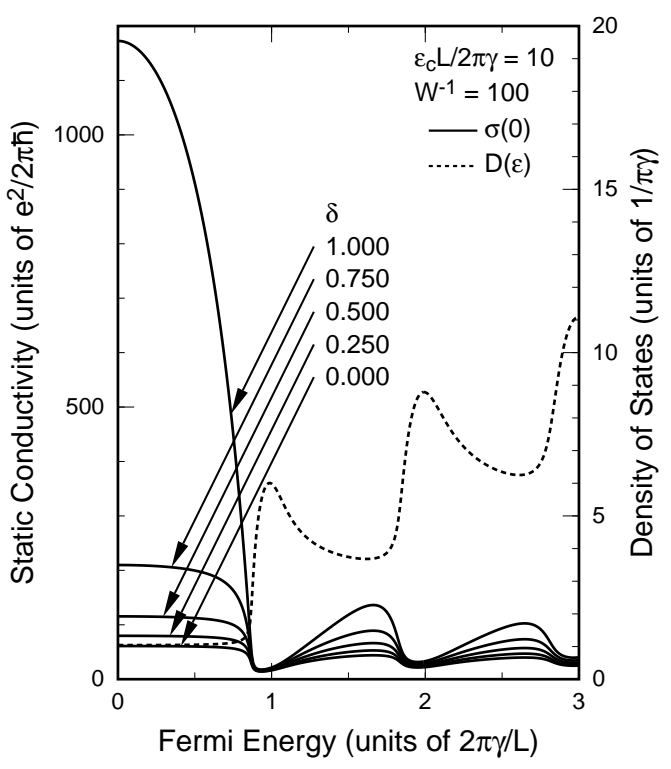

Fig. 3. The static limit of the dynamical conductivity as a function of the Fermi energy. The solid lines represent $\sigma(0)$ and the dashed line the density of states.

ductivity exhibits a sharp peak but the peak value corresponding to the static conductivity remains finite.

Figure 3 shows $\sigma(0)$ as a function of the Fermi energy $\varepsilon_{F}$ together with the density of states at $\varepsilon_{F}$. It shows that the exceptionally sharp Drude peak occurs when the Fermi level lies in the energy range where only metallic bands with linear dispersion exist. Except in the energy range $-2 \pi \gamma / L \lesssim \varepsilon_{F} \lesssim+2 \pi \gamma / L$, the static conductivity exhibits clear oscillation related to the density of states, which is essentially same as that obtained by Boltzmann transport equation for model short-range scatterers [37].

The conductance is given by the transmission probability between eigenstates in infinitely long ideal leads with exactly same energy. The absence of backscattering and the perfect conductance occur only between these eigen states in an ideal system. In the dynamical conductivity describing optical absorption, however, transitions occur between eigen states in the presence of scatterers causing mixtures of different bands. It is reasonable, therefore, that the dynamical conductivity is broadened and becomes different from $\delta(\omega)$, although the conductance remains ideal due to the special feature of the absence of backward scattering. The absence of backscattering is expected to lead to a di- vergent conductivity in the static limit, however.

A quantum correction to the conductivity gives important information on the behavior of this dynamical conductivity. The lowest order correction is known to be given by so-called maximally crossed diagrams. The result for $\varepsilon_{F}>2 \pi \gamma / L$ is given by [39]

$$
\delta \sigma \approx \frac{2 e^{2}}{\pi \hbar} \frac{1}{\alpha} \frac{L_{\phi}}{\sqrt{i \omega \tau_{\phi}+1}}, \quad D=\left(\alpha^{-1}-1\right) v_{0}^{2} \tau
$$

where $\tau$ is the relaxation time, $\tau_{\phi}$ is the inelastic relaxation time, $v_{0}=\gamma / \hbar$, and

$$
\alpha=1-\frac{\sum_{n}\left[1-\left(2 \pi \gamma n / \varepsilon_{F} L\right)^{2}\right]^{1 / 2}}{\sum_{n}\left[1-\left(2 \pi \gamma n / \varepsilon_{F} L\right)^{2}\right]^{-1 / 2}} .
$$

This shows that the static conductivity $(\omega=0)$ is proportional to the phase coherence length $L_{\phi}=\sqrt{D \tau_{\phi}}$ qualitatively in agreement with the numerical result discussed above and that the dynamical conductivity exhibits a divergence $\propto \omega^{-1 / 2}$ for $\tau_{\phi} \rightarrow \infty$. Quantitatively, however, this lowest quantum correction is likely to be insufficient.

\section{Summary and Conclusion}

In summary, a brief review has been given on electronic and transport properties of carbon nanotubes mainly from a theoretical point of view. In the effective-mass scheme electrons in nanotubes are regarded as neutrinos on cylinder surface with a fictitious Aharonov-Bohm flux passing through the cross section. The $\mathbf{k} \cdot \mathbf{p}$ description is particularly useful for revealing extraordinary properties of metallic nanotubes. In fact, in metallic carbon nanotubes, there is at least a single channel transmitting through the system without backscattering independent of energy for scatterers with potential range comparable to or larger than the lattice constant. This channel is sensitive to inelastic scattering when several bands coexist at the Fermi level, however. This has been demonstrated in a model that an electron looses its coherence for a distance determined by a phase coherence length. The dynamical conductivity exhibits a singular dependence corresponding to the presence of a perfect conducting channel. 


\section{Acknowledgments}

The author acknowledges the collaboration with $\mathrm{H}$. Suzuura. This work was supported in part by Grantsin-Aid for Scientific Research and for COE (12CE2004 "Control of Electrons by Quantum Dot Structures and Its Application to Advanced Electronics") from Ministry of Education, Science and Culture in Japan. Numerical calculations were performed in part using the facilities of the Supercomputer Center, Institute for Solid State Physics, University of Tokyo.

\section{References}

[1] H. Ajiki and T. Ando, J. Phys. Sco. Jpn. 62 (1993) 1255 .

[2] S. N. Song, X. K. Wang, R. P. H. Chang, and J. B. Ketterson, Phys. Rev. Lett. 72 (1994) 697.

[3] J. E. Fischer, H. Dai, A. Thess, R. Lee, N. M. Hanjani, D. L. Dehaas, and R. E. Smalley, Phys. Rev. B 55 (1997) R4921.

[4] M. Bockrath, D. H. Cobden, P. L. McEuen, N. G. Chopra, A. Zettl, A. Thess, and R. E. Smalley, Science 275 (1997) 1922.

[5] L. Langer, V. Bayot, E. Grivei, J. -P. Issi, J. P. Heremans, C. H. Olk, L. Stockman, C. Van Haesendonck, and Y. Bruynseraede, Phys. Rev. Lett. 76 (1996) 479.

[6] A. Yu. Kasumov, I. I. Khodos, P. M. Ajayan, and C. Colliex, Europhys. Lett. 34 (1996) 429.

[7] T. W. Ebbesen, H. J. Lezec, H. Hiura, J. W. Bennett, H. F. Ghaemi, and T. Thio, Nature (London) 382 (1996) 54.

[8] H. Dai, E.W. Wong, and C. M. Lieber, Science 272 (1996) 523.

[9] A. Yu. Kasumov, H. Bouchiat, B. Reulet, O. Stephan, I. I. Khodos, Yu. B. Gorbatov, and C. Colliex, Europhys. Lett. 43 (1998) 89.

[10] A. Fujiwara, K. Tomiyama, H. Suematsu, M. Yumura, and K. Uchida, Phys. Rev. B 60 (1999) 13492.

[11] S. J. Tans, M. H. Devoret, H. Dai, A. Thess, R. E. Smalley, L. J. Geerligs, and C. Dekker, Nature (London) 386 (1997) 474.

[12] S. J. Tans, R. M. Verschuren, and C. Dekker, Nature (London) 393 (1998) 49.

[13] D. H. Cobden, M. Bockrath, P. L. McEuen, A. G. Rinzler, and R. E. Smalley, Phys. Rev. Lett. 81 (1998) 681.
[14] S. J. Tans, M. H. Devoret, R. J. A. Groeneveld, and C. Dekker, Nature (London) 394 (1998) 761.

[15] A. Bezryadin, A. R. M. Verschueren, S. J. Tans, and C. Dekker, Phys. Rev. Lett. 80 (1998) 4036.

[16] J. Tersoff, Appl Phys. Lett. 74 (1999) 2122.

[17] M. P. Anantram, S. Datta, and Y.-Q. Xue, Phys. Rev. B 61 (2000) 14219.

[18] K. -J. Kong, S. -W. Han, and J. -S. Ihm, Phys. Rev. B 60 (1999) 6074.

[19] H. J. Choi, J. Ihm, Y. -G. Yoon, and S. G. Louie, Phys. Rev. B 60 (1999) R14009.

[20] T. Nakanishi and T. Ando, J. Phys. Sco. Jpn. 69 (2000) 2175.

[21] J. Kong, E. Yenilmez, T. W. Tombler, W. Kim, and H. J. Dai, R. B. Laughlin, L. Liu, C. S. Jayanthi, and S. Y. Wu, Phys. Rev. Lett. 87 (2001) 106801.

[22] P. R. Wallace, Phys. Rev. 71 (1947) 622.

[23] J. C. Slonczewski and P. R. Weiss, Phys. Rev. 109 (1958) 272.

[24] M. V. Berry, Proc. Roy. Soc. London A392 (1984) 45.

[25] T. Ando, T. Nakanishi, and R. Saito, J. Phys. Sco. Jpn. 67 (1998) 2857.

[26] T. Ando and T. Nakanishi, J. Phys. Sco. Jpn. 67 (1998) 1704.

[27] T. Nakanishi and T. Ando, J. Phys. Sco. Jpn. 68 (1999) 561.

[28] P. L. McEuen, M. Bockrath, D. H. Cobden, Y. -G. Yoon, and S. G. Louie, Phys. Rev. Lett. 83 (1999) 5098.

[29] A. Bachtold, M. S. Fuhrer, S. Plyasunov, M. Forero, E. H. Anderson, A. Zettl, and P. L. McEuen, Phys. Rev. Lett. 84 (2000) 6082.

[30] H. Suzuura and T. Ando, Physica E 6 (2000) 864.

[31] H. Suzuura and T. Ando, Mol. Cryst. Liq. Cryst. 340 (2000) 731.

[32] H. Suzuura and T. Ando, Phys. Rev. B 65 (2002) 235412 .

[33] See for example, W. A. Harrison, Electronic Structure and the Properties of Solids (W.H. Freeman and Company, San Francisco, 1980).

[34] C. L. Kane and E. J. Mele, Phys. Rev. Lett. 78 (1997) 1932.

[35] T. Ando and H. Suzuura, J. Phys. Soc. Jpn. 71 (2002) 2753.

[36] H. Akera and T. Ando, Phys. Rev. B 43 (1991) 11676.

[37] T. Seri and T. Ando, J. Phys. Sco. Jpn. 66 (1997) 169.

[38] T. Ando, J. Phys. Soc. Jpn. 71 (2002) 2505.

[39] H. Suzuura and T. Ando, unpublished 